\title{
Light-scattering measurements of micro-deformations in granular materials
}

\author{
M. Erpelding ${ }^{1}$, A. Amon ${ }^{1, a}$, and J. Crassous ${ }^{1}$ \\ Institut de Physique de Rennes (IPR) - UMR 6251 - Campus de Beaulieu, Bât 11 A, 35042 Rennes \\ Cedex, France
}

\begin{abstract}
Diffusive Wave Spectroscopy is used to measure very small strains of the order of $10^{-5}-10^{-3}$. That method gives a new full-field measurement of the strain at the vicinity of the surface of highly scattering materials. The principle of the method is presented and application to the measurement of micro-deformations in a granular material are shown.
\end{abstract}

\section{Introduction}

The developpement of full-field measurements of strain is still an expanding area of research which has led to optical, non intrusive methods such as Digital Image Correlation or interferometric techniques (speckle or holographic interferometry) [1,2]. Such optical methods provide a map of the deformation at the surface of a sample or in a plane embedded in a transparent sample [3]. We propose a new full-field optical method based on Diffusive Wave Spectroscopy [4,5]. The originality of our method compared to others is : first that the method is not merely surfacic but scan a layer in the vicinity of a surface, and second that the range of deformation probed, $10^{-5}-10^{-3}$ is very small.

Our method is of use for highly light scattering samples, which the case of a lot of common materials such as Téflon, plaster, ceramics, stintered glass, paper, PVC, wood, silicon seal or polyurethane foam. When such diffusive materials are illuminated with a laser source, a speckle pattern arises from interferences of the different optical paths followed by light inside the material. It is important to underline that the effect is not a mere surface effect : the phase differences are acquired in the bulk of the sample. In the case of backscattered light, the typical extension of the random walk followed by photons is small enough to consider that only small volumes are explored. We have shown [6] that in this configuration a spatially resolved decorrelation map can be obtained, directly linked to the local strain in the vicinity of the surface. Because the speckle pattern arises from a multiple diffusion process, which implies long paths for the light, very small strains are sufficient to yield a decorrelation. Hence our method provides a new kind of full field measurement of strain in the range $10^{-5}$ to $10^{-3}$. Such a technique gives a spatially resolved measurement of the deformation either in configurations for which a heterogeneous repartition of strain is awaited, as we will show in the following, or when heterogeneities are likely to appear, namely in the case of apparition of fractures.

In this paper, we first present the principle of measurement, and demonstrate its use on an elastic material (a slab of Téflon submitted to a small strain). Then we apply the method to a material for which the mechanical response is not well-known : a granular material.

\footnotetext{
a e-mail: axelle.amon@univ-rennes1.fr
} 


\section{Principle of the measurement}

\subsection{General principle}

When light propagates in strongly scattering materials, light rays are submitted to successive deviations so that a part of the light is scattered while the directly transmitted light is strongly attenuated even in the absence of absorption. The typical length characterizing that diffusive process is called the transport mean free path, $l^{*}$. This length characterizes the elementary step of the random walk followed by a light ray inside the media. Example of materials giving rise to such high scattering process have been given in the introduction. A typical value of $l^{*}$ is $260 \mu \mathrm{m}$ for Téflon.

When such a scattering media is enlighten with coherent light, the backscattered light field results from the interferences of numerous rays having followed different paths inside the material and consequently with different phases. The interference pattern obtained by such a random process is called speckle. Contrary to most actual speckle methods which are based on surface reflection, in our case rays propagate inside the sample. More precisely in the backscattering configuration the optical rays probe a volume of typical size $\left(l^{*}\right)^{3}$ in the vicinity of the illuminated surface, as shown schematically on Figure 1(a).

If the scatterers are displaced, the speckle pattern changes, a complete decorrelation occuring for a variation of the total typical optical path length the order of the wavelength of light. As paths are very long in a diffusive process, a displacement of the scatterers all along the optical path of only a fraction of the wavelength is sufficient to lead to a decorrelation. Consequently, the measurable strains are very small. Information processing with speckle patterns is based on the calculation of correlation between two patterns corresponding to two different states of the material. Decorrelation gives a measurement of the amount of change in the speckle pattern between two states, which can be linked to the internal changes of the position of the scatterers in the sample. In our case different states correspond to different loadings of a sample.

\subsection{Practical implementation}

The principe of our method is schematized on Figure 1(a). The illuminated plane side of the sample is imaged on a CCD camera. A polarizer ensures us that only strongly backscattered light is collected by selecting only depolarized light. A diaphragm allows us to control the size of the speckles on the camera. This size $\left(l_{c}\right.$ on Figure 1(b)) has been chosen to optimize the use of the pixels of the camera [7]. The images thus obtained are divided into metapixels containing $N \sim 200$ speckle spots. Ensemble averages over each of these areas are then performed, giving one value of correlation for each metapixel. One point of the final correlation map is one metapixel, i.e. one large square on figure 1(b).

The transversal lens magnification $\gamma$ is chosen so that the dimension $l^{*}$ on the object corresponds to a metapixel on the image. As a matter of fact, as the typical extension of the random walk of a ray in the sample is $l^{*}$, a natural averaging takes place over this size by the diffusive process. This effect gives a natural limitation to the resolution of the method.

\subsection{Theoretical background}

A theoretical description of the multiple scattering process can be performed in order to link the measured correlation function to the elementary displacements of the scatterers in the sample [6]. The correlation function of the electric field calculated between two states (1) and (2) of the sample is :

$$
g_{E}^{(12)}=\frac{\left\langle E_{1} E_{2}^{*}\right\rangle}{\left\langle\left|E_{1}\right|\right\rangle\left\langle\left|E_{2}\right|\right\rangle}
$$

where $E_{i}$ is the electric field in the state $(i)$ and $\langle\cdot\rangle$ represents an ensemble average over several speckle spots. Using the diffusion approximation [5,8], this correlation function can be written [9]:

$$
g_{E}^{(12)}=\int_{s} P(s)\left\langle\exp ^{j \Delta \phi_{s}(1,2)}\right\rangle d s,
$$


(a)

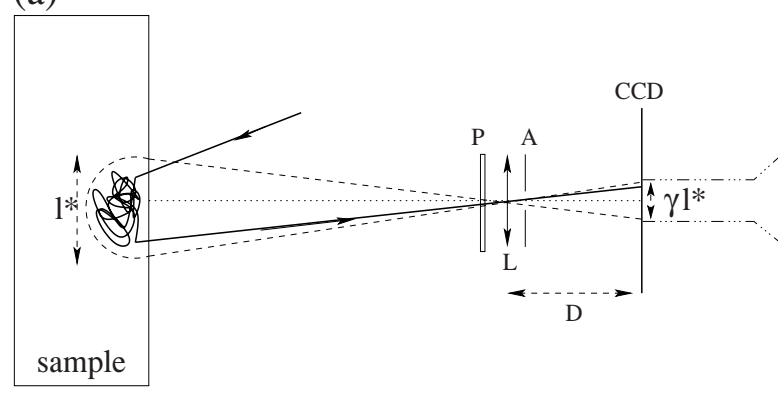

(b)

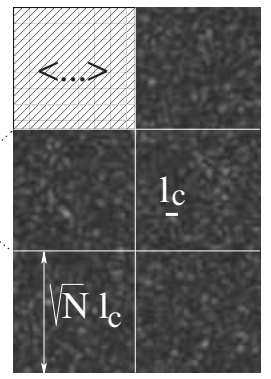

Fig. 1. (a) Schematic of the measurement. An example of ray path is shown in solid line. The lens L images the front of the sample on a CCD camera. $\mathrm{P}$ is a polarizer and A a diaphragm aperture. (b) Speckle image: $l_{c}$ is the typical size of the coherence areas. Ensemble averages performed done over squares containing typically $N$ speckles. The optical setup is chosen so that $\gamma l^{*}=\sqrt{N} l_{c}$.

where $\Delta \phi_{s}(1,2)$ is the phase difference for an optical path of length $s$ between the states (1) and (2) and contains the information about the motion of the scatterers. $\langle\cdot\rangle$ represents an average over all paths of length s. $P(s)$ is the path length distribution, and depends on the optical properties of the materials and on the geometry of the experiment [8]. The calculation of $\Delta \phi_{s}(1,2)$ implies a description of the elementary displacements of the scatterers which can be linked to the strain tensor $\mathbf{U}[6]$. In the multiple scattering limit, the distribution $P(s)$ can be computed from the diffusion equation and $\left\langle\exp ^{j \Delta \phi_{s}(1,2)}\right\rangle$ can be expressed in terms of the isotropic invariants of the strain tensor [10]. The expression of the correlation function is then [6]:

$$
\left|g_{E}^{(12)}\right| \approx \exp \left(-\eta k l^{*} \sqrt{3 f(\mathbf{U})}\right)
$$

where:

$$
f(\mathbf{U})=\frac{1}{15}\left(\sum_{i} U_{i i}\right)^{2}+\frac{2}{15} \sum_{i, j} U_{i j}^{2}
$$

In equation (3), $k$ is the wave vector of the light field $: k=2 \pi / \lambda$ with $\lambda$ the wavelength of light in the medium, $\eta$ is a numerical factor of order unity ranging from 1.5 to 2.7 and depending on the polarisation states of the illuminating and the scattered light [9]. Numerical coefficients in equation (4) come from a calculation in the case of Mie scatterers dispersed in a homogeneous matrix and may not be true for a heterogeneous material of unknown internal structure. Nevertheless, we will use this expression for all materials in first approximation [6].

Practically, in experiments, correlations are calculated over intensities and not amplitudes. These two quantities are linked via the Siegert relation [4] so that the intensity correlation function calculated as:

$$
G_{I, \text { mes }}^{(12)}=\frac{\left\langle I_{1} I_{2}\right\rangle-\left\langle I_{1}\right\rangle\left\langle I_{2}\right\rangle}{\sqrt{\left\langle I_{1}^{2}\right\rangle-\left\langle I_{1}\right\rangle^{2}} \sqrt{\left\langle I_{2}^{2}\right\rangle-\left\langle I_{2}\right\rangle^{2}}},
$$

verified $G_{I, \text { mes }}^{(12)} \approx \exp \left(-2 \eta k l^{*} \sqrt{3 f(\mathbf{U})}\right)$. Consequently, for an incompressible sample, the logarithm of the decorrelation is directly proportionnal to the elastic energy accumulated in the material in comparison to a reference state.

\section{Demonstration of the method}

\subsection{Experimental set-up}

We present results obtained in a plane-stress geometry for a sample of Téflon (polytetrafluoroethylene, PTFE). We measured the transport mean free path for that material $: l^{*} \simeq 260 \mu \mathrm{m}$. In the following, 
we use a tabulated value of the Young's Modulus : $E=500 \mathrm{MPa}$ and consider the material to be incompressible : Poisson's ratio $v \simeq 0.5$. The size of the sample is $4 \mathrm{~cm} \times 3 \mathrm{~cm}$. The configuration is chosen in order to yield a heterogeneous strain field in a plane stress geometry: the Téflon sample is $5 \mathrm{~mm}$ thick and forces are applied ponctually with a blade larger than the thickness of the sample in order to keep a uniform applied stress in the direction orthogonal to our observation plane. To apply a force we use a vibrator (Brüel\&Kjær4810) and we deduce the applied force from the current input into the vibrator.

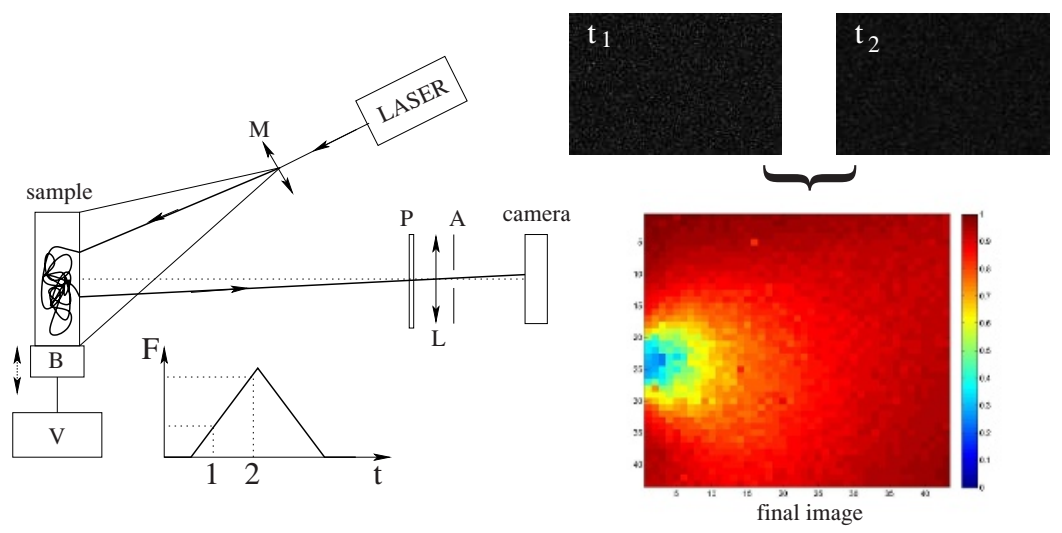

Fig. 2. Schematic of the experimental set-up. $M$ is a microscope objective, $\mathrm{V}$ the vibrator, $\mathrm{B}$ the blade, $\mathrm{P}$ the polarizer, L the lens, A the diaphragm aperture. A schematic graph of the force applied with the blade as a function of time is shown. Two extracts of speckles images and the corresponding calculated correlation map are shown. The color scale of the correlation maps will be the same on all the Figures and is indicated on the figure.

A schematic of the experimental set-up is shown on figure 2. We use a Ventus continuous-wave linearly polarized laser of wavelength $532 \mathrm{~nm}$ and maximal power $\sim 50 \mathrm{~mW}$. The beam emerging from the laser source is expended by a microscope objective of magnification $\times 10$. The beam incident on the sample is sufficiently wide so that the intensity is roughly spatially homogeneous on the imaged surface. The camera is a DALSA PT-41-04M60 of $2352 \times 1728$ resolution and pixel size $7.4 \mu \mathrm{m}$. The magnification of the lens $\gamma \simeq 0.5$ is obtained by choosing a focal length $f^{\prime}=100 \mathrm{~mm}$ for the lens $\mathrm{L}$ and a distance $D=150 \mathrm{~mm}$ between the lens $\mathrm{L}$ and the CCD of the camera. The diaphragm aperture has a diameter $d \simeq 3.6 \mathrm{~mm}$. Typically, the final map after average will give $58 \times 43$ metapixels for the final decorrelation map.

\subsection{Point load: Boussinesq profile}

Typical experimental maps obtained are shown on the upper line of Figure 3(c) for different values of the exerted force corresponding to a ramp of lineary increasing then decreasing force. The values of the forces corresponding to the successive images are : $0 \mathrm{~N}, 2.7 \mathrm{~N}, 8.1 \mathrm{~N}, 2.7 \mathrm{~N}$ and $0 \mathrm{~N}$.

The sample is large enough to compare the strain maps obtained experimentally to the analytical Boussinesq response for a point load on a semi-infinite plane [11]: isostress lines are then circles tangentials to the loading point. Qualitatively, the profiles are in good agreement with the awaited spatial repartition of the strain and the recorrelation is quite accurate when the force goes back to a previous value in the reverse cycle.

On Figure 3(c), under each experimental result are displayed calculated maps obtained by a fitting procedure described in [6]. As a matter of fact, using the Boussinesq analytical solution and equation 5, 


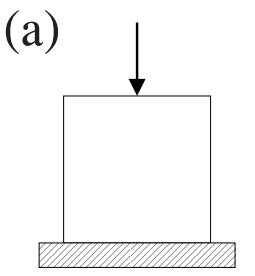

(c)
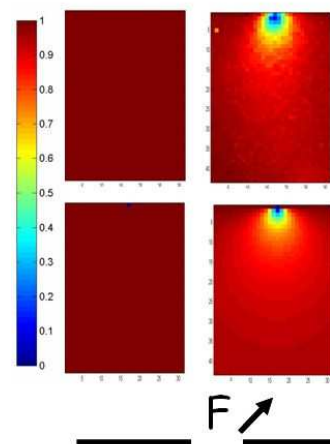

(b)

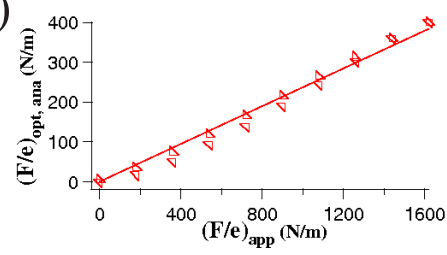

Téflon
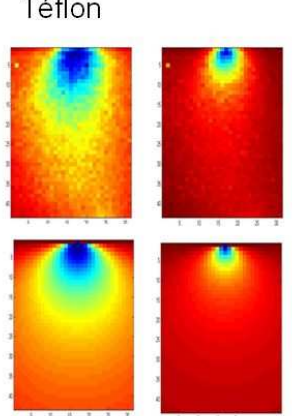

$19 \mathrm{~mm}$
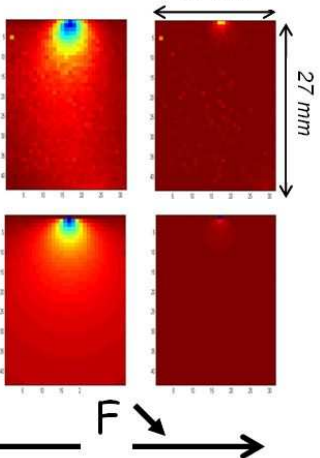

Fig. 3. Point load configuration, (a) the load is applied with a blade, a null displacement is imposed at the opposite side of the sample ; (b) comparison of the force applied experimentally divided by the thickness of the sample with the value obtained by the best fit with eq. 6 . The slope of the linear fit is $0.24 ;$ (c) upper line : experimental decorrelation maps ; bottom line : best analytical fit with eq. 6

the theoretical correlation function can be explicitely calculated as :

$$
G_{I}^{(12)}=\exp \left(-\frac{F}{e} 4 \eta k \sqrt{\frac{2}{15}} \frac{\sqrt{3\left(1+2 v^{2}\right)}}{\pi E} \frac{z}{x^{2}+z^{2}}\right)
$$

¿From is expression, it is possible to exhibit an unique fitting parameter : $\frac{F}{e}$. It is then possible to find the value of the parameter minimizing a measurement of the difference between the experimental and the theoretical map. Figure 3(b) gives the value of the parameter obtained from the fitting procedure as a function of the same parameter deduced from the experiment. Reversibility and linearity are well verified. The slope of the linear fit is 0.24 . Such a result is rather good taking into account several points extensively discussed in reference [6]. First, several parameters in equation 6 are empirical and large errors are intrisic to the measurement of $l^{*}$. Secondly, the internal structure of Téflon is not known and the Mie theory may not be accurate to describe the diffusive process in that medium. Finally, the finite size of the system should be taken into account. Indeed, we have shown that a better agreement is obtained when using a numerical description of the complete geometry in the case of a material for which Mie diffusion holds [6].

\section{Mechanical response of a granular material}

We have used our method to study the mechanical response of a granular material submitted to a localized load. The experimental set-up is shown on Figure 4. We used granular samples made of spherical glass beads (silibead) of diameter in the range $70-110 \mu \mathrm{m}$. The beads were placed in a metallic cell of inner dimensions $(46 \times 36 \times 15) \mathrm{mm}^{3}$ with glass walls. The samples were always prepared following the same procedure. A cylindrical tip of diameter $10 \mathrm{~mm}$ was fixed onto the vibrator. We measure the force applied to the sample with a force sensor (FUTEK FSH02665) inserted between the vibrator 


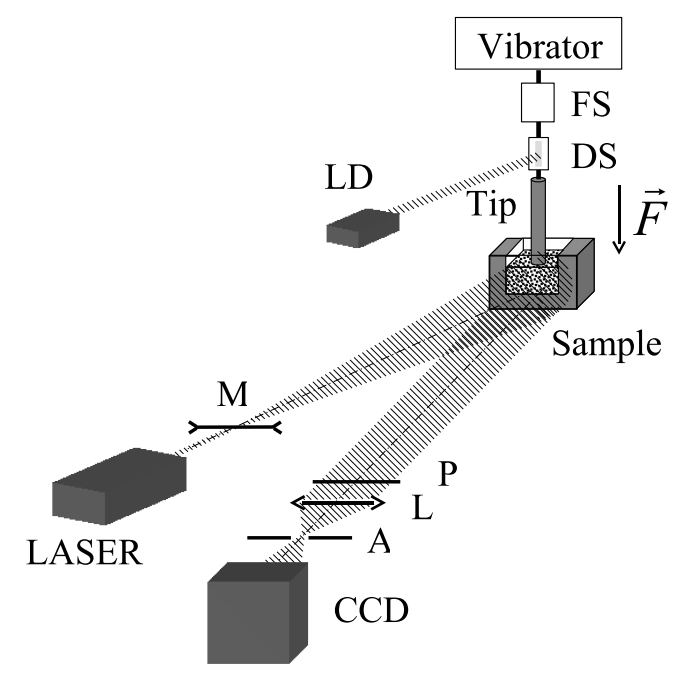

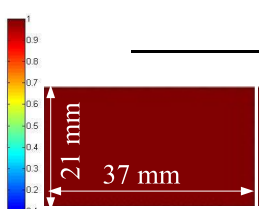

(a)

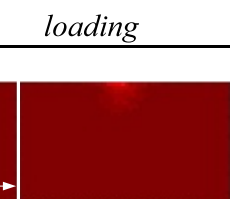

(b)

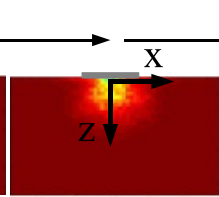

(c)

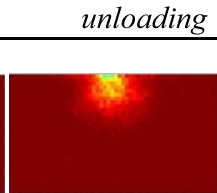

(d)

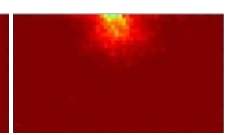

(e)

Fig. 4. Up : Experimental setup. (M), (L), (A) and (P) same as figures 1 and 2. The sample is deformed by a tip mounted on a vibrator. The applied force and the tip displacement are measured using a force sensor (FS) and a displacement sensor (DS) coupled with a laser diode (LD). Bottom : Experimental correlations maps obtained for the cyclic loading of a granular sample. Correlations are computed with respect to the same reference image corresponding to an applied force $F=3.79 \cdot 10^{-2} \mathrm{~N}$. The force increments between each map and the reference map are (a) $\Delta F=0 \mathrm{~N}$, (b) $\Delta F=2.76 \cdot 10^{-2} \mathrm{~N}$, (c) $\Delta F=5.33 \cdot 10^{-2} \mathrm{~N}$, (d) $\Delta F=2.43 \cdot 10^{-2} \mathrm{~N}$, and (e) $\Delta F=0 \mathrm{~N}$.

and the tip. We also measure the displacement of the tip using a photodiode (Hamamatsu S5629-01 Position Sensitive Detector). The decorrelation maps obtained for the first loading-unloading cycle are shown on Figure 4.

Using our measurement on a Téflon sample as a reference, we can compare the response of the granular material to the response of an elastic sample [12]. Figure 5 shows the correlation maps computed between the reference image and the image corresponding to the maximum applied force. For selected pixels indicated on the maps, we analyze the variation of $\ln \left(g_{I}\right)$ as a function of the force increment $\Delta F$. For the Teflon sample, we find that $\ln \left(g_{I}\right)$ is linear with $\Delta F$, as expected for a linear elastic sample. In contrast, for the granular sample, our results clearly show a nonlinear behavior of $\ln \left(g_{I}\right)$ with $\Delta F$. We are currently studying how that non linearity is affected with the number of applied loading cycles.

\section{Conclusion}

We have presented a new full-field measurement of the strain at the vicinity of the surface of scattering materials. The method derives from Diffusive Wave Spectroscopy and yields the measurement of a quantitative map of the strain repartition in the vicinity of the surface of a scattering sample. The use of a scattering process makes it possible to probe very small strains : $10^{-3}$ to $10^{-5}$, beyond most of the usual strain measurement methods. Because of the smallness of the range fo strains measured, applications to brittle and fragile materials are promising. Indeed, precursors of fractures should be 

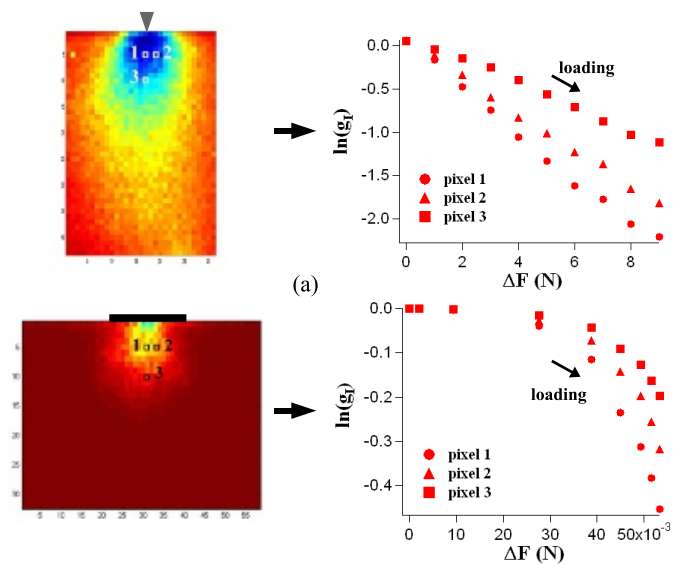

(b)

Fig. 5. Examples of correlation maps $g_{I}(x, z)$ obtained for Teflon (a) and granular (b) samples for given values of $\Delta F$. The position and size of the tips are indicated on top of the images. For the three pixels indicated on each map, we plot $\ln \left(g_{I}\right)_{\text {pixel }}$ as a function of the force increment $\Delta F$ during the first loading.

accessible by this method. As a first application we have presented first measurements of the mechanical response of a granular material to a localized force, evidencing nonlinearity of the deformation as a function of the applied force.

\section{References}

1. Photomechanics, Topics in Applied Physics 77, edited by P. K. Rastogi (Springer, 2000).

2. F. Hild S. Roux, Strain, 42, (2006), 69-80.

3. G. Restivo G. L. Cloud, Exp. Mech., 48, (2008) 731-740.

4. B. J. Berne and R. Pecora, Dynamic Light Scattering With Applications to Chemistery, Biology, and Physics (Dover Publication Inc., 2000).

5. D.J. Pine, D.A. Weitz, J.X. Zhu and E.Herbolzheimer, J. Phys. France, 51, (1990) 2102.

6. M. Erpelding, A. Amon J. Crassous, Phys. Rev. E, 78, (2008) 046104.

7. V. Viasnoff, F. Lequeux, and D. J. Pine, Review of Scientific Instruments, 73, (2002) 2336.

8. A. Ishimaru, Wave Propagation and Scattering in Random Media, (Academic Press, 1978).

9. Dynamic Light Scattering : The Method and Some Applications, edited by W. Brown (Oxford University Press, 1993).

10. J. Crassous, Eur. Phys. J. E, 23, (2007) 145-152.

11. L. Landau and E. Lifchitz, Théorie de l'élasticité (Mir, Moscou, 1967).

12. M. Erpelding, A. Amon J. Crassous, submitted to Europhysics Letters. 